\title{
THREE-DIMENSIONAL TIME RESOLVED MEASUREMENTS OF CHARGED QUANTUM DOTS IN NANOCONFINED CHANNELS USING TOTAL INTERNAL REFLECTION FLUORESCENCE MICRSCOPY (TIRFM)
}

\author{
T. Wynne and S. Pennathur \\ University of California, Santa Barbara, CA, USA
}

\begin{abstract}
In this work, we use total internal reflection fluorescence microscopy (TIRFM) to excite $\mathrm{CdSe} / \mathrm{ZnS}$ core-shell quantum dots (QD) in $100 \mathrm{~nm}$ deep fused silica fluidic channels. Since the channels are shallower than the evanescent wave depth we can continuously probe the QD dynamics within the electrostatic environment of our system. We systematically perform experiments at varying salt concentrations to gain insight about the electrostatic behavior of finite sized charged particles in confined nanochannel environments. First, we describe the governing equation of electrostatics in confined environments. Next, we show successful TIRF measurements in both nanofluidic channels as well as unconfined coverslips. Finally, we compare transverse distributions of quantum dots in both environments for various background salt concentrations. Our data shows that as the electric double layer thickness increases, the particle is repelled from the wall by repulsive electrostatic interactions between the particle and the wall, and confinement shows increasingly different behavior than the traditional Poisson Boltzmann theoretical approach may suggest.
\end{abstract}

\section{INTRODUCTION}

In the last few years, nanofluidics has emerged as its own field for the lab-on-chip community due to the unique coupling physics that occur at the nanometer length scale. For example, nanofluidic channels have been shown to have better separation efficacy, concentration ability, and new modalities for biomolecule manipulation and interrogation [1] Nanofluidics can also be used to study biomolecules in confined environments that are on the length scale of in-vivo biological environments. Here, both the surfaces of the system as well as the surfaces of the biomolecules are important in governing the behavior of the system. Such studies will not only give us insight towards the effects of confinement in biomolecular systems, but will also serve as a design tool for the nanofluidic biomolecular analytical devices.

To date, there have been many theoretical and experimental studies of small ions in confined nanofluidic systems, with excellent agreement between theory and experiment [2, 3]. However, only few experimental and theoretical address finite size particles within nanofluidic channels, where the size of the particle can significantly change the background potential distribution. Although many molecular dynamics and density functional theory (DFT) models simulations have been performed in ion channels, nanofluidics for lab on chip systems generally relies on fluidic channels that are between $50-100 \mathrm{~nm}$ [1]. In terms of larger sized channels, Gillespie [4], recently presented analytic theory for dilute colloids in charged slits, and Boy et al. [5] are developing a computational model that describes the electrostatics and Brownian motion of large (finite-sized) particles of arbitrary geometry confined in nanochannels using non-graded adaptive grids and level set methods to represent interfaces.

In this work, we propose to use a novel imaging technique, Total Internal Reflectance Fluorescence Microscopy (TIRF) to experimentally interrogate $17 \mathrm{~nm}$ quantum dots in $100 \mathrm{~nm}$ confined nanofluidic channels. Total internal reflection fluorescence microscopy (TIRF) is a powerful analysis tool that exploits the evanescent wave produced by total internal reflection to excite fluorescent particles within a few hundred nanometers of a surface. Generally, TIRF has been used for imaging biological samples at the surface, since it gives superior signal to noise ratios as it does not excite background fluorescence [6], but since the advent of microfluidics [7], TIRF has been used to study fundamental properties of microfluidic systems near the surface. For example, Guasto and Breuer [8] used TIRF to probe near-wall velocimetry in microfluidic channels ( 40 microns) to measure fluidic slip in Poiseuille flows with a variety of tracer particles, high-speed near wall measurements of QDs with TIRF in Poiseuille flows [9], and Sadr et al. studied near wall measurements of electro-osmotic flow in rectangular driven flow [10]. All microchannel TIRF studies have tracking times limited to a few milliseconds because the fluorescent tracers diffuse transversely out of the evanescent wave illumination region.

Therefore, although microchannel TIRF has allowed for great insight into the composition of surfaces in terms of the slip length, this technique has not been used to probe the electrostatic environment of the surface. Here we confine QDs in nanoscale fluidic channels that do not allow the evanescent wave to fully decay before reaching the far wall, as seen in Figure 1a. This enables us to study QDs for long periods of time (on the order of 20s) and use intensity distributions to characterize how finite sized charged particles behave in buffers of different ionic strengths.

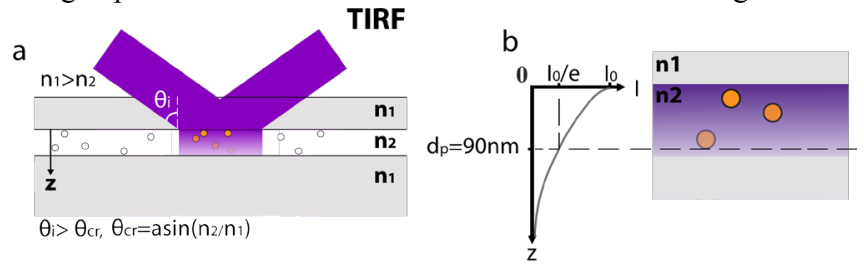

Figure 1: Total internal reflection (TIR) schematic (a) and evanescent wave exponential decay intensity profile (b). (a) TIR occurs when light reaches a sharp dielectric interface of decreasing index of refraction with an angle greater than the critical angle $\theta_{c}$ as defined in Snell's law. (b) An exponentially decaying wave is transmitted into the less dense medium. TIRF uses this light to excite fluorescence within a few hundred nanometers of the surface. In this study, we use channels that do not allow the evanescent wave to fully decay which enables us to continuously excite QDs with a non-uniform excitation field in $z$.

\section{GOVERNING EQUATIONS}

To solve for the electrostatic distribution of ions in a nanofluidic channels, we assume that the distribution of species $i$ will follow the Boltzmann distribution

$$
c_{i}=c_{\infty, i} \exp \left(-\frac{Z_{i} e \psi(z)}{k_{B} T}\right)
$$

where $c_{\infty}$ is the concentration $(\mathrm{mol} / \mathrm{L})$ in the bulk, $\psi$ is the potential, $e$ is the elementary charge, $k_{B}$ is the Boltzmann constant, 
$T$ is the temperature, $Z$ is the valence number, $n_{\infty}$ is the number density $\left(\mathrm{m}^{-3}\right)$ in the bulk, and $z$ is the distance from the wall. This distributions allows us to write the governing equation for electrostatics as follows [11]:

$$
\frac{d^{2} \psi}{d z^{2}}=-\frac{e}{\varepsilon} \sum_{i=1}^{N} Z_{i} n_{\infty, i} \exp \left(-\frac{Z_{i} e \psi(z)}{k_{B} T}\right)
$$

This nonlinear Poisson Boltzmann equation is then solved subject to the boundary conditions at the solid-liquid interface.

To solve for Dirichlet boundary condition of zeta potential, we use a self-consistent charge and triple layer model following that of Wang [3]. First, the chemistry at the silica wall is governed by 3 reaction equations:

$$
\begin{aligned}
& \mathrm{SiOH} \underset{\leftarrow}{\mathrm{SiO}} \mathrm{O}^{-}+\mathrm{H}^{+}, \quad K_{-}=\frac{c_{H S} \Gamma_{-}}{\Gamma_{0}}
\end{aligned}
$$

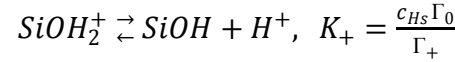

$$
\begin{aligned}
& \mathrm{SiO}^{-}+\mathrm{M}^{+} \underset{\leftarrow}{\rightarrow} \mathrm{SiOM}, \quad K_{M}=\frac{\Gamma_{\mathrm{M}}}{\mathrm{C}_{\mathrm{Ms}} \Gamma_{-}}
\end{aligned}
$$

where $K_{i}$ is the known reaction constant for each reaction equation, $\Gamma$ is the number of available sites and $M$ is a metal cation species that can adsorb to the silica surface. Next, the total number of available sites is conserved, and surface charge is defined by the difference between the number of positive and negatively charged sites at the silica surface. Finally, one can relate the potential drop across each layer with $C_{i}$ being the Stern capacitance between triple layer planes:

$$
\psi_{0}-\psi_{\beta}=\frac{\sigma_{0}}{C_{1}}
$$

Furthermore, we can eliminate the surface charge ad solve directly for zeta potential as a function of concentration by using Grahame's equation as follows:

$$
\sigma_{0}=\sqrt{2 \varepsilon k_{B} T}\left(\sum_{i=1}^{N} n_{\infty, i}\left[\exp \left(-\frac{Z_{i} e \zeta}{k_{B} T}\right)-1\right]\right)^{\frac{1}{2}}
$$

where $\sigma$ is the surface charge and $\zeta$ is the potential at the slip plane. Similar relations must be applied to the QD, which has a carboxyl coating with a specified charge distribution. Note that a significant parameter used to define our systems is the Debye length, $\lambda_{D}$, which is the characteristic length scale for the thickness of the electric double layer (EDL). The Debye length decreases for higher ionic strengths and scales as $n_{\infty}^{-1 / 2}$

$$
\lambda_{D}=\left(\frac{\varepsilon k T}{e^{2} \sum_{i=1}^{n} z_{i}^{2} n_{\infty, i}}\right)^{\frac{1}{2}}
$$

Non-zero zeta potentials on the wall result in a non-uniform hydronium ion distribution and hence a spatial $p \mathrm{H}$ gradient across the channel for large Debye lengths. The zeta potential can therefore be used as a boundary condition for finding the correct $p \mathrm{H}$ at various transverse positions in the channel and the average $p \mathrm{H}$ in the channel can be defined by:

$$
\langle p \mathrm{H}\rangle_{i}=-\log _{10}\left(\frac{\int_{0}^{h} d z c_{i} c_{H}}{\int_{0}^{h} d z c_{i}}\right)
$$

\section{MATERIALS AND METHODS}

\section{Buffer and Sample Preparation}

Boric acid crystals (EMD, BX0865-1) were dissolved in filtered ultra-pure deionized water (Millipore, Milli-Q) to create $200 \mathrm{mM}$ ionic strength stock solution. We monitor $p \mathrm{H}$ (Oakton, $p \mathrm{H}$ 11 ) and titrate all buffer solutions with sodium hydroxide solution (Fisher Scientific, S318-500) to $p \mathrm{H} 9.15$ then dilute to 5, 10, 20 and $50 \mathrm{mM}$ ionic strengths. Due to confinement in the nanochannel, it has been shown that the $p \mathrm{H}$ in a nanochannel will be lower than the bulk $p \mathrm{H} \mathrm{[12],} \mathrm{the} \mathrm{effect} \mathrm{is} \mathrm{significant} \mathrm{at} \mathrm{lower}$ ionic strengths Since the intensity of QD emission is very sensitive to $p \mathrm{H}$ [13], we further titrate the solutions to corrected $p \mathrm{H}$ values according to Eqs. 1-7. This is an iterative process due the coupling between total ionic strength (which increases during titration) and the corrected $p \mathrm{H}$, resulting in set of buffer concentrations corresponding to that shown in Table 1.

We use water-soluble CdSe/ZnS core-shell quantum dots with carboxyl polymer coating (Invitrogen; model\# A10200) with core diameter of $\sim 7 \mathrm{~nm}$ and overall hydrodynamic diameter of $\sim 17 \mathrm{~nm}$. The peak wavelength for emission is centered at $625 \mathrm{~nm}$ with FWHM of $30 \mathrm{~nm}$. We dilute the stock $8 \mu \mathrm{M}$ QD solution down to $10 \mathrm{pM}$ in sodium-borate buffer at $p \mathrm{H} 9.15$ for coverslip experiments and 50pM in $p \mathrm{H}$ corrected sodium-borate solutions for channel experiments. We chose these dilutions of QDs to be able to resolve individual quantum dots for coverslip measurements $(10 \mathrm{pM})$ and isolate single QDs in the channel for confined experiments $(50 \mathrm{pM})$.

Table 1: Corrected $p \mathrm{H}$ values for nanofluidic confined TIRF

\begin{tabular}{|c|c|c|c|}
\hline $\boldsymbol{\lambda}_{\mathbf{D}}$ & Borate [C] & $\mathbf{N a O H}[\mathbf{C}]$ & Corrected $\boldsymbol{p H}$ \\
\hline $4.29 \mathrm{~nm}$ & $5 \mathrm{mM}$ & $3.05 \mathrm{mM}$ & 9.35 \\
\hline $3.04 \mathrm{~nm}$ & $10 \mathrm{mM}$ & $5.67 \mathrm{mM}$ & 9.27 \\
\hline $2.15 \mathrm{~nm}$ & $20 \mathrm{mM}$ & $10.9 \mathrm{mM}$ & 9.20 \\
\hline $1.35 \mathrm{~nm}$ & $50 \mathrm{mM}$ & $23.3 \mathrm{mM}$ & 9.15 \\
\hline
\end{tabular}

studies. Column 1 - Debye length, defined by Eq. 6, Column 2 Borate buffer concentration, Column 3 - sodium-hydroxide ionic strength, Column 4 - corrected $p \mathrm{H}$ based on Eqs. 1-7

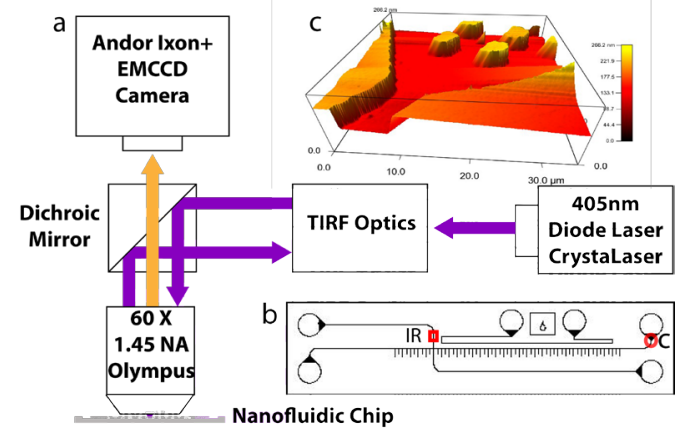

Figure 2: (a) Schematic of the home built TIRF system. (b) Layout of the nanofluidic chip. The interrogation region (IR) is in the topleft channel $2 \mathrm{~mm}$ before the intersection. (c) AFM of the $100 \mathrm{~nm}$ etched fused silica features.

\section{Nanochannel Fabrication and substrates}

Nanofluidic channels are fabricated on $525 \mu \mathrm{m}$ thick, $100 \mathrm{~mm}$ diameter fused silica wafers (Hoya Corp.; model\# 4W55-325-15CSTD) using contact lithography (EVGroup, EValign 620) with positive tone photoresist (Shipley, 3217M) and NF3 plasma dry etch (Applied Materials, AMT 8100) to create 100nm deep rectangular trenches, AFM viewgraph in Figure 2c (Asylum Research, MFP-3D). $1.8 \mathrm{~mm}$ access holes are CNC-drilled into a $170 \mu \mathrm{m}$ thick, $100 \mathrm{~mm}$ diameter quartz wafer (Montco Silicon; Lot\# 4QUARTZ/B). Both wafers are RCA cleaned before fusion bonding at $1050 \mathrm{C}$. The wafer is diced into $12 \times 50 \mathrm{~mm}$ nanofluidic chips with pattern in Figure $2 b$.

\section{Experimental Setup}

TIRF illumination is created from home built system (Figure 2a) that includes a $405 \mathrm{~nm} 50 \mathrm{~mW}$ continuous wave diode laser (CrystaLaser TL405-050), the beam is directed with free space optics (ThorLabs, Newport) that feed into the laser port of an upright microscope (Olympus BX41) with QD optimized fluorescence filters (Chroma; Z405RDC, HQ620/25m) and an oil immersion objective lens (Olympus; 60x TIRF, 1.45 NA). The laser beam is expanded and focused off axis at the back focal plane of a 1.45 NA objective lens to produce a collimated beam incident at $\sim 70$ degrees from the normal for total internal reflection. The 
emitted fluorescence from the QDs is captured on a backilluminated EMCCD (Andor, Ixon+).

\section{EXPERIMENTAL PROCEDURE \\ Coverslip TIRF}

Approximately $5-10 \mathrm{~mm}$ wide, $170 \mu \mathrm{m}$ thick quartz coverslips are sonicated in acetone then isopropyl alcohol for 5 minutes then rinsed with ultra-pure deionized water and dried with nitrogen before each experiment. For each buffer concentration, we pipette $5 \mu \mathrm{L}$ of $10 \mathrm{pM}$ QD solution onto a $1 \mathrm{~mm}$ thick glass slide (Corning, 2974-3x1) and cover with a second $170 \mu \mathrm{m}$ thick coverslip. Each experiment measures the intensity of 1 to 15 different QDs at a time that diffuse in and out of the evanescent wave, due to the $\sim 3 \mathrm{~ms}$ residence time of each particle.

\section{Confined FTIRF}

For these frustrated TIRF (FTRIF) experiments, our experimental procedure is as follows. First, we fill the nanochannel with DI water by capillary action. All subsequent flushing steps are preformed using a $7.1 \mathrm{kV} / \mathrm{m}$ electric field applied to the top-left channel (see Figure 2). First, we flush with DI water to remove any bubbles in the channel. Next, the channel is electrokinetically flushed with $100 \mathrm{mM} \mathrm{NaOH}$ for 15 minutes, which removes surface contaminants by etching the surface slightly. The channel is then flushed with DI water for 30 minutes. After cleaning, $p \mathrm{H}$ corrected sodium-borate buffer solution is electrokinetically driven into the channel and allowed to equilibrate in the channel for at least 30 minutes to ensure chemical equilibrium of the EDL structure. Finally, we replace the buffer in the top-left well with $p \mathrm{H}$ corrected 50pM QD of the same buffer ionic strength and drive them into the channel until we can isolate one in the region of interest (Figure 2) with the camera. We then focus the objective and acquire 1500 frames at a $3 \mathrm{~ms}$ exposure time, at $82 \mathrm{~Hz}$ and with 300EM gain. Note that in these experiments, only one QD is imaged at a time.

\section{Data Reduction}

The images are converted to 16 bit TIFF files and processed with Blair and Dufresne [14] PIV functions using commercial software (MATLAB, inc) to locate the particle. We sum a $3 \times 3$ array around a peak corresponding to a QD to count all of the light emitted from the QD, then subtract noise (based on the average noise for 9 pixels), and normalize with the flat field based on the $\mathrm{x}$ $y$ coordinates of the peak. We perform this operation for every frame and find the corresponding distribution of measured intensities. Figure 3 shows an example of a distribution for $10 \mathrm{mM}$ buffer concentration in a confined $100 \mathrm{~nm}$ channel, which also shows the phenomena of quantum dot coagulation and blinking. Figure 3 (a) shows a "normal" single QD distribution whereas (b) shows a QD cluster distribution. Some histograms appeared to be heavily influenced by QD blinking or clusterings, and therefore were omitted from the averaged distributions over all experiments.

\section{RESULTS}

\section{Coverslips}

For a baseline, we first measure unconfined intensity distributions of $10 \mathrm{pM}$ QD solutions between a fused silica coverslip and glass slide which are separated by a distance much larger than the evanescent wave penetration depth. The normalized intensity distributions are shown in Figure 4a for 5, 10 and 20mM sodium-borate buffer at $p \mathrm{H} 9.15$ along with a fitted curve. The data shows that QD concentration near a charged surface increases with increased buffer concentration, Figures $4 \mathrm{c}$,d,e show example images at 5, 10 and 20mM respectively. The negatively charged

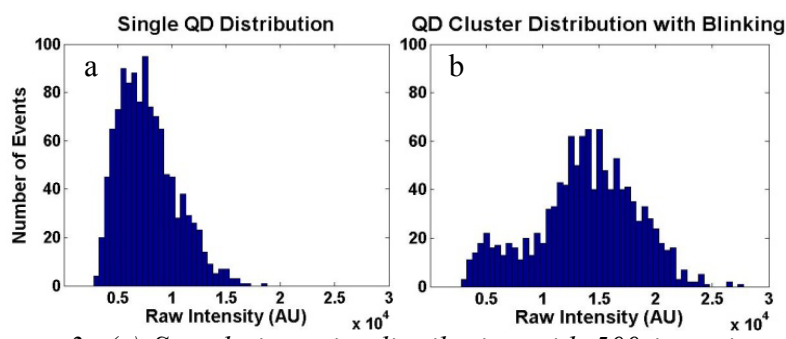

Figure 3: (a) Sample intensity distribution with 500 intensity unit bins, for a single confined $Q D$ in $10 m M$ sodium-borate buffer. (b) Sample intensity distribution of a $Q D$ cluster, seen by the high mean intensity. There is also with significant blinking characterized by the secondary peak.

particles have repulsive interactions with the negatively charged walls, thus for longer $\lambda_{\mathrm{D}}$, the QDs distribution will be closer to the wall. Figure $4 \mathrm{~b}$ shows the corresponding theoretical small ion potential distribution for a flat wall [11] for the same concentrations/conditions, which qualitatively agrees to the coverslip data. Note that the experimental data has a peak in concentration (as opposed to the theoretical distributions and exponential fits) because of the low probability that particles will have trajectories that are barely above the detection limits.
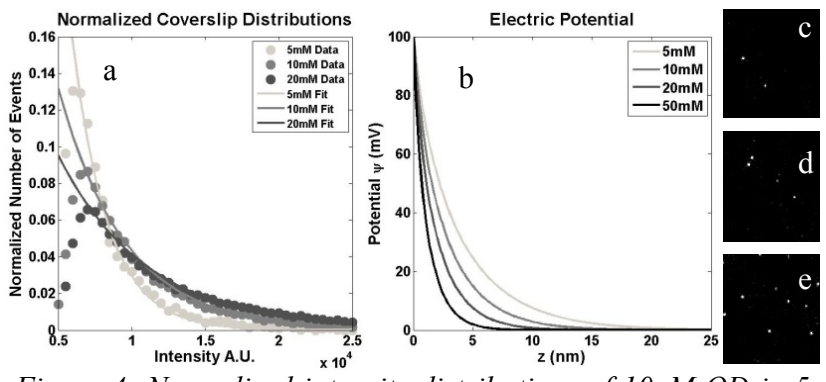

Figure 4: Normalized intensity distributions of 10pM QD in 5, 10 and $20 \mathrm{mM}$ sodium-borate buffer. The curves show that the $Q D$ density distribution is lowest near the surface for $5 \mathrm{mM}$ and increases with increased buffer concentrations. Sample images are show in (c) $5 \mathrm{mM}$ (d) $10 \mathrm{mM}$ and (e) $20 \mathrm{mM}$. These show that the near surface QD density is higher for higher buffer concentrations, indicating the length scale of the repulsive force is shorter.
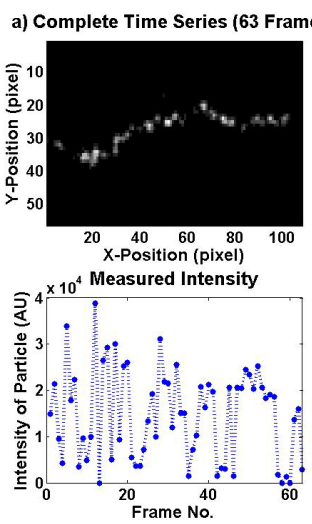
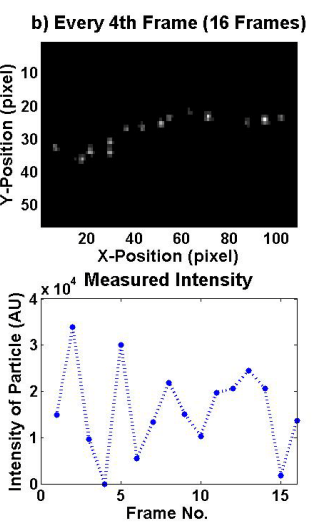

Figure 5: Demonstration of confined TIRF showing superposed frames of a single $Q D$ electrokinetically driven with $1.56 \mathrm{kV} / \mathrm{m}$ applied electric field in a 100nm channel. (a) Top: Compilation of 63 frames superposed, Bottom: measured intensity (A.U.) of the $Q D$ in each corresponding frame. (b) Top,Bottom: same data as (a) but analyzing every $4^{\text {th }}$ frame for visual clarity. Images acquired at $64.5 \mathrm{~Hz}$ with $3 \mathrm{~ms}$ exposure time and $300 \mathrm{EM}$ gain. 


\section{Nanofluidic Confined Experiments}

First, we demonstrate confined TIRF by showing a time series of a single quantum dot kinetically driven with $1.56 \mathrm{kV} / \mathrm{m}$ electric field in a $100 \mathrm{~nm}$ deep channel in Figure 5. In this figure we show 64 consecutive frames of a single electrokinetically driven QD in a nanofluidic channel for 1 second, traveling (from left to right) a total distance of $108 \mathrm{um}$. Although the particle is completely confined within the illumination depth, the quantum dot is not detected in all frames due to blinking, which is a property exhibited by QDs where they enter a "dark state" do not emit fluorescence for a random period of time [13]. In any case, this figure shows that we can study quantum single quantum dots with TIRF over much longer time scales than previously capable [11].

To compare QD intensity distributions in confinement for different electrostatic environments we analyze 900-1100 (out of 1500 frames) intensity values for a single QD diffusing by pure hindered Brownian motion over $\sim 18$ seconds. Note that the time for $17 \mathrm{~nm}$ QDs to diffuse across the $100 \mathrm{~nm}$ depth of the channel is on the order of $1 \mathrm{~ms}$, and therefore the $3 \mathrm{~ms}$ exposure intensity measurements quantify detected photons from the QD over multiple oscillations through the channel in the $\mathrm{z}$ dimension. Figure 6 shows the normalized intensity distributions of confined single quantum dots for different ionic strengths. Note that since we accounted for the change in $p \mathrm{H}$ within the channels, we can assume the quantum dots have the same emission response for all ionic strengths. Therefore, our data shows that the intensity peak of the distribution is greater for lower ionic strengths. This suggests that for higher ionic strengths, the quantum dot samples a larger range of the channel (and therefore an average intensity that is smaller) than the QDs in lower ionic strengths. We suggest that this result is due to the effect of thicker double layers, and results in a broader distribution.

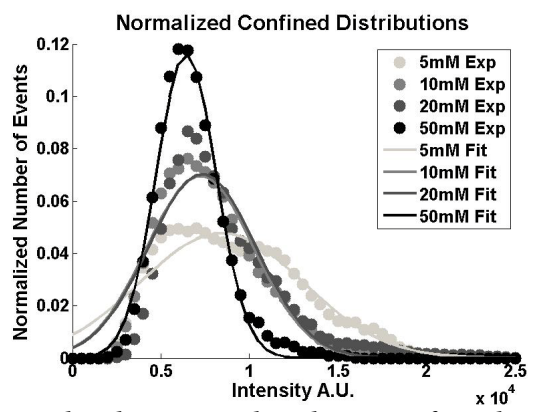

Figure 6: Normalized intensity distributions of single quantum dots confined in a $20 \mu \mathrm{m} \times 100 \mathrm{~nm}$ fused silica channel. The distributions show that the peak intensity is higher for lower ionic strengths. This suggests that for higher ionic strengths the QD samples a larger range of channel resulting in a lower average intensity than at low ionic strength where the $Q D$ samples a smaller range due to repulsion at the walls.

The differences of the confined distributions and the coverslips distributions can also give us insight to the effect of confinement on finite sized particles. For example, the $5 \mathrm{mM}$ experiment is significantly broader in confined nanochannels, suggesting that confinement repels the quantum dot further than in the coverslip case. This makes sense because the channel walls each contribute electrostatic repulsion due to the double layer, effectively confining the quantum dots to a smaller region of the channel, whereas in coverslips quantum dots are free to diffuse to infinity in the transverse dimension. These measurements are the first such fundamental measurements to quantify confinement effects of finite size particles in nanoconfined channels.

\section{DISCUSSION}

We demonstrate the confined TIRF with quantum dots using $100 \mathrm{~nm}$ fused silica channels with a model for necessary $p \mathrm{H}$ corrections due to confinement. We present unconfined distributions that qualitatively agree with PB theory, and compare these with confined distributions which show some deviation at lower ionic strengths. We attribute this to the fact that the confined environment must force particles to be near the center of the channel, whereas unconfined particles are free to diffuse large lengths scale away from the wall. These studies are the first fundamental studies of finite sized charged particles within confined channels, and may lead to breakthroughs in nanofluidic separation studies and understanding of fundamental biomolecule behavior in confined channels.

\section{ACKNOWLEDGEMENTS}

The authors thank Jesper Toft Kristensen and Kristian Lund Jensen for their numerical calculations for nanochannel $p \mathrm{H}$ corrections. This work is supported by grant from the Institute for Collaborative Biotechnologies (ICB) and UCSB startup fund. The fabrication was completed at UCSB, a National Nanotechnology Infrastructure Network (NNIN) member.

[1] M. Napoli, J.C.T. Eijkel, and S. Pennathur, "Nanofluidic technology for biomolecule applications: a critical review". Lab on a Chip, 10, 8 (2010).

[2] S. Pennathur and J.G. Santiago, "Electrokinetic transport in nanochannels. 2. Experiments". Anal. Chem., 77, 21 (2005).

[3] M. Wang and A. Revil, "Electrochemical charge of silica surfaces at high ionic strength in narrow channels". Journal of Colloid and Interface Science, 343, 1 (2010)

[4] D. Gillespie, "Analytic Theory for Dilute Colloids in a Charged Slit". The J. of Phys. Chem. B, 114, 12 (2010)

[5] D. Boy, et al., "Particle Separation by Capillary Electrophoresis in Nanochannels", IMA Microfluidics: Electrokinetic and Interfacial Phenomena. 2009.

[6] D. Axelrod, T. Burghardt, and N. Thompson, "Total Internal Reflection Fluorescence". Ann. Rev. Biophys. Bioengr., 93, 13 (1984).

[7] T. Squires and S. Quake, "Microfluidics: Fluid physics at the nanoliter scale". Rev. Mod. Phys., 77, 3 (2005).

[8] P. Huang, J. Guasto, and K. Breuer, "Direct measurement of slip velocities using three-dimensional total internal reflection velocimetry". J. Fluid Mech., 566 (2006)

[9] J. Guasto and K. Breuer. "Micro-Velocimetry using timeresolved measurements of quantum dots in a microchannel". ECI International Conference on Heat Transfer and Fluid Flow in Microscale. Whistler (2008).

[10] R. Sadr, et al., "An experimental study of electro-osmotic flow in rectangular microchannels". J. Fluid Mech., 506 (2004)

[11] R. Hunter, Foundations of Colloid Science. Oxford: Oxford Science Publication. 1993

[12] D. Bottenus et al., "Experimentally and theoretically observed native $\mathrm{pH}$ shifts in a nanochannel array". Lab on a Chip, 8, 9 (2008).

[13] N. Durisic, et al., "A Common Mechanism Underlies the Dark Fraction Formation and Fluorescence Blinking of Quantum Dots". ACS Nano, 3, 5(2009).

[14] D. Blair and E. Dufresne. "The Matlab Particle Tracking Code Repository". http://physics.georgetown.edu/matlab/. 